\title{
Recubrimientos micro/nanoestructurados de aleaciones ligeras mediante proyección fría para la protección y reparación de componentes de elevado valor añadido: Estado del arte ${ }^{(\cdot)}$
}

\author{
J. Bedoya*, N. Cinca* y J.M. Guilemany*
}

\begin{abstract}
Resumen
La técnica de Proyección Fría (Cold Gas Spray - CGS) ha atraído considerablemente la atención de los investigadores en la última década dadas sus ventajas respecto a otras técnicas convencionales de proyección térmica, como la obtención de recubrimientos de altísima densidad y sin degradación (ya sea oxidación o cambios de fase), además de su gran potencial a nivel económico y ambiental por ser un proceso más eficiente y respetuoso con el medio ambiente. Este estudio presenta una revisión bibliográfica respecto a la obtención de recubrimientos micro/nanoestructurados base aluminio mediante proyección fría sobre sustratos de aleaciones ligeras, el cual permitirá tener una visión global sobre la viabilidad de utilizar la proyección fría como técnica para la protección, recuperación y restauración de componentes de elevado valor añadido, especialmente aquéllos fabricados con aleaciones ligeras (base aluminio y magnesio) los cuales, por su baja densidad y propiedades mecánicas, son ampliamente utilizados en diferentes sectores de la industria como el transporte, la aeronáutica y generación de energía.
\end{abstract}

Palabras clave Proyección fría (CGS); Aleaciones ligeras; Recargues; Soldadura fuerte; Recubrimientos.

\section{Micro/nanostructured coatings of light alloys by cold spray for surface protection and repair of high value-added components: State of the art}

Abstract

Keywords

\begin{abstract}
The Cold Gas Spray - CGS technique has greatly attracted the attention of the researchers in the last decade due to advantages compared to other conventional thermal spray processes. It presents outstanding characteristics such as high density coatings and absence of thermal degradation (oxidation or phase transformation). In addition, CGS is an efficient and green technology. This paper presents a bibliographic review related to micro/nanostructured aluminium-based coatings of by CGS on light alloy substrates. It provides an overview of the feasibility of using the Cold Gas Spray as a technique for the protection, recovery and repair of high value-added components, especially those made of light alloys (aluminium and magnesium base) which, by their low density and mechanical properties, are widely used in several industrial sectors such as transport, aerospace and power generation.
\end{abstract}

Cold Gas Spray (CGS); Light alloys; Weld overlay; Brazing; Coatings.

\section{INTRODUCCIÓN}

Las tecnologías de proyección térmica forman parte de la Ingeniería de Superficies. Éstas permiten obtener recubrimientos con propiedades específicas para satisfacer una necesidad industrial concreta (resistencia a oxidación, corrosión, desgaste, requisitos de dureza e incluso biocompatibilidad, entre otras $)^{[1-4]}$.

La característica común de las tecnologías de proyección térmica convencionales es el aporte de energía cinética y energía térmica al material de recubrimiento, normalmente en forma de polvo o varilla. El material de partida normalmente se funde total o parcialmente y se proyecta sobre una superficie limpia donde impacta e interacciona mecánicamente e incluso metalúrgicamente con el sustrato. Las características del recubrimiento dependen esencialmente del proceso de proyección térmica escogido, de los parámetros de proyección y del material a proyectar ${ }^{[5 \text { y } 6]}$.

Las técnicas se clasifican en función del origen e intensidad de las energías aportadas, básicamente, combustión y arco eléctrico. En la figura 1 se representan las distintas técnicas en función del rango de temperatura y velocidad de las partículas.

(•) Trabajo recibido el día 9 de junio de 2012 y aceptado en su forma final el día 5 de octubre de 2012.

* Centre de Projecció Tèrmica (CTP). Universitat de Barcelona. Martí i Franqués 1, E-08028, Barcelona, Spain. E-mail: jbedoya@cptub.eu. 


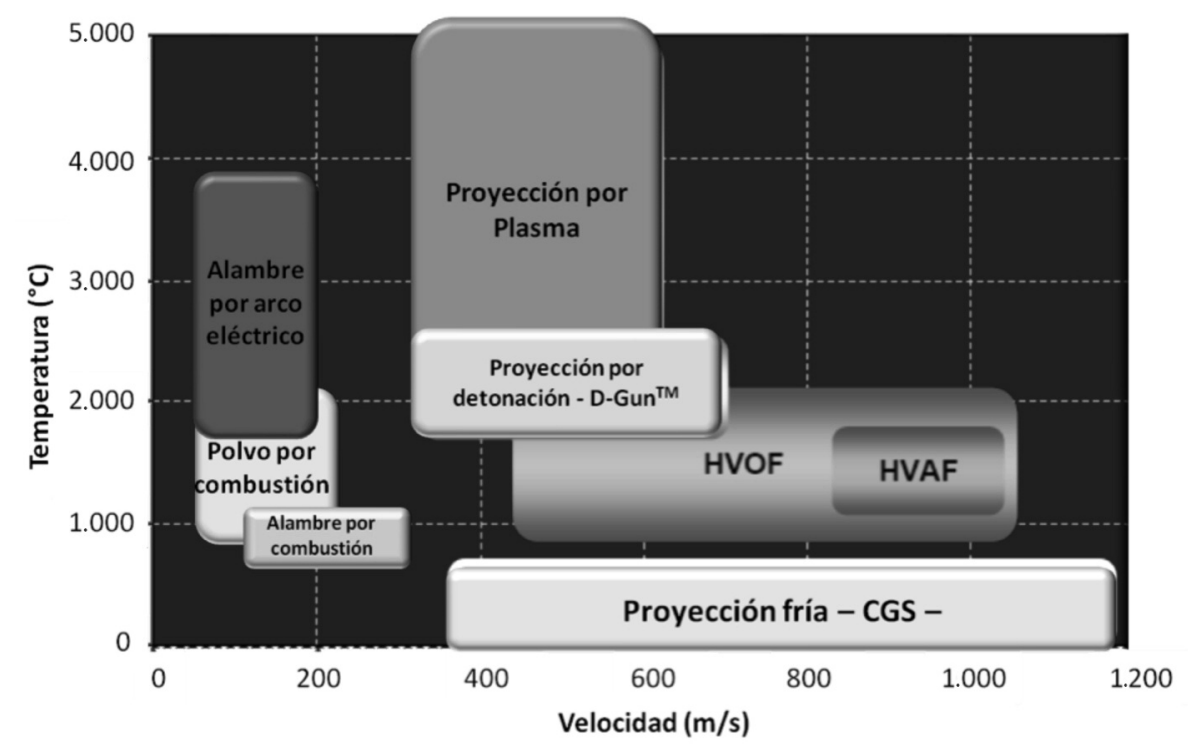

Figura 1. Clasificación de las tecnologías de proyección térmica según el rango de temperatura y velocidad de las partículas.

\section{Figure 1. Classification of thermal spray technologies by the temperature and velocity of sprayed particles.}

En comparación con otros métodos de modificación superficial, la proyección térmica presenta una gran versatilidad en cuanto a los materiales que pueden proyectarse y los sustratos o superficies que se pueden utilizar. Prácticamente todos los materiales que funden sin descomponerse dando una fase líquida estable, se pueden utilizar para formar recubrimientos por proyección térmica convencional. Aunque el material proyectado llegue al sustrato fundido o parcialmente fundido, el sustrato no se expone a altas temperaturas, por lo cual su forma y características estructurales y mecánicas no cambian, a diferencia de lo que sucede en soldadura y recargues por soldadura, técnicas en las cuales se genera una zona afectada por el calor (ZAC).

\section{LA PROYECCIÓN FRÍA (COLD GAS SPRAY, CGS)}

Uno de los desarrollos más recientes en las tecnologías de proyección térmica es la proyección fría ${ }^{[7}$ y 8$]$. En esta técnica el material proyectado no llega a fundir, manteniéndose en estado sólido al llegar al sustrato. El material de recubrimiento, en forma de polvo, se inyecta en un haz de gas inerte y caliente a alta presión, que le confiere energía cinética y cierto aporte térmico, acelerándolo para impactar sobre el sustrato a velocidad supersónica. La figura 2 muestra un esquema del proceso. En las técnicas convencionales, el paso de las partículas por el estado líquido puede resultar en oxidaciones tanto del sustrato como del recubrimiento. Estos óxidos pueden disminuir los valores de adherencia, tanto adhesiva como cohesiva, del recubrimiento. La técnica de proyección fría evita estas reacciones, permitiendo proyectar materiales metálicos altamente reactivos.

Las partículas se deforman plásticamente al impactar sobre el sustrato, adhiriéndose al mismo, formando el recubrimiento, por lo que los materiales más dúctiles son los más favorables ${ }^{[9}$ y 10$]$. Este es un proceso complejo, ya que la deformación plástica de las partículas que llegan al sustrato va a depender, en términos generales, de su comportamiento a altas velocidades de deformación. Así, cuanto más duro sea el sustrato, más se deformarán las primeras partículas que llegan, produciéndose un mayor aplastamiento de la misma. La deformación de la segunda capa de deposición vendrá influenciada por el estado de las partículas depositadas en la primera capa y no por el sustrato, es decir, por sus propiedades mecánicas y la posibilidad de que hayan sufrido endurecimiento por deformación.

Al impactar las partículas, toda la energía cinética se convierte en energía de deformación plástica y calor disipado. Dependiendo del par sustrato-partículas se podrán distinguir una serie de regiones con deformación plástica severa, cristales fuertemente texturados, granos recristalizados e incluso, según algunos autores, microestructuras solidificadas a distancias menores a $1 \mu \mathrm{m}$ de la interfase $\mathrm{e}^{[11]}$. 


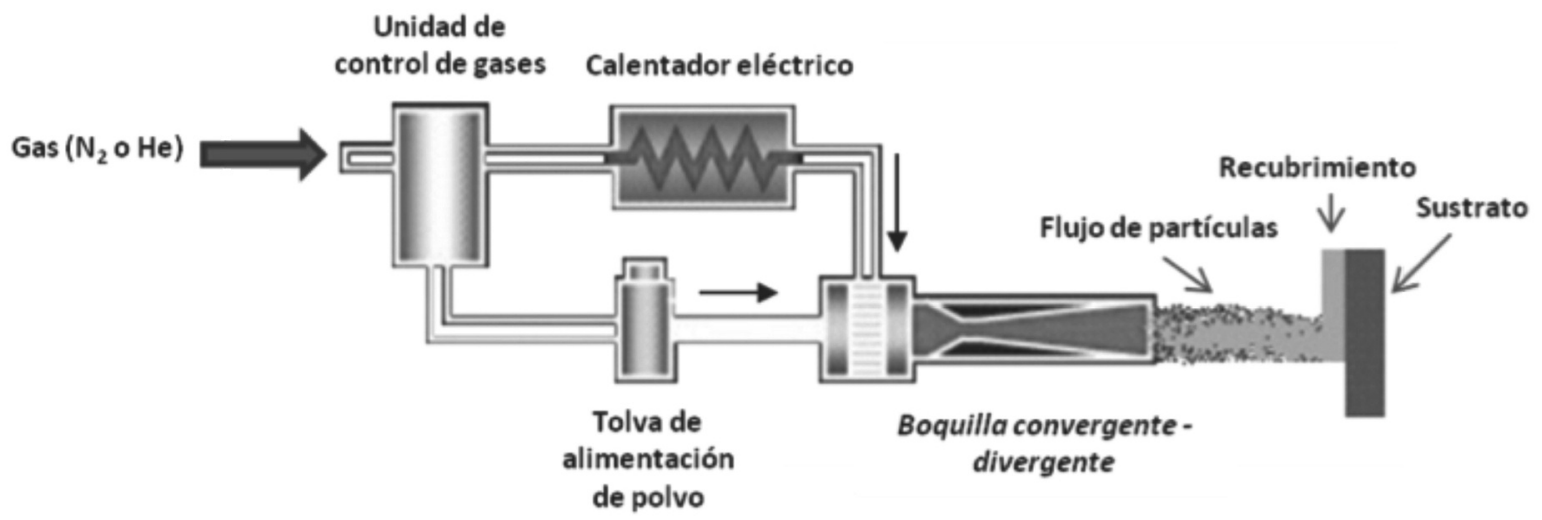

Figura 2. Esquema del proceso de proyección fría - CGS.

Figure 2. Schematic representation of Cold Gas Spray system.

Las partículas que no se hayan adherido al sustrato durante la primera deposición, habrán producido cráteres que, a su vez, modificarán la actividad del sustrato para facilitar la adhesión; por lo tanto, también habrá unos parámetros de tipo geométrico, tales como superficie de área de contacto, ancho y profundidad del cráter, que deberán ser tenidos en cuenta para conseguir recubrimientos con las propiedades deseadas.

Uno de los parámetros claves del proceso de proyección fría es la velocidad de las partículas previa al impacto sobre el sustrato. Para una deposición adecuada se requiere, dependiendo del material, una cierta velocidad mínima o crítica para que haya adhesión, tal como se ilustra en la figura 3; este valor depende significativamente de las propiedades termomecánicas del polvo y del sustrato, morfología y tamaño de partícula ${ }^{[12-14]}$. Por debajo de esta velocidad crítica, las partículas que impactan generan solamente una erosión del sustrato ${ }^{[5}$ y 6 ; por encima, la deposición de las partículas mejora hasta un máximo que, en algunos casos, puede superar el $95 \%$.

Por otro lado, la velocidad de la partícula, para un material dado, vendrá determinada por el diseño de la pistola de proyección utilizada, la presión y la temperatura del gas transportador ${ }^{[15}$ y 16$]$ e incluso el tipo de gas utilizado ${ }^{[17]}$. Hay que tener también presente que la cantidad de deformación plástica que se disipará en forma de calor será mayor para mayores velocidades de deformación ${ }^{[18]}$ y que esta particularidad influenciará notablemente tanto a la adhesión de las partículas proyectadas como a la estructura final $y$, por ende, a las propiedades conseguidas. Assadi et al. ${ }^{[19]}$ señalan que el ablandamiento térmico producido por el calor desprendido adiabáticamente junto con la inestabilidad de cizalladura de la interfase son las variables que juegan un papel principal en los procesos microscópicos, dando lugar a una unión de par- tículas satisfactoria; ello va a conducir a una ventana estrecha de condiciones de proyección donde este proceso va a tener lugar. El estudio de los mecanismos de unión que se producen es complejo ya que va a depender, a su vez, de las propias variables del proceso. Así, para el rango de velocidades con las que se proyectan las partículas, se producen dos procesos que son competitivos: endurecimiento por deformación y ablandamiento por el calor generado por fricción en la interfase.

Este modo de deposición permite conservar, al menos parcialmente, las características micro y nanoscópicas del polvo original. La figura 4 muestra un recubrimiento de aluminio sobre acero de baja aleación obtenido en el Centro de Proyección Térmi$\mathrm{ca}^{[20]}$

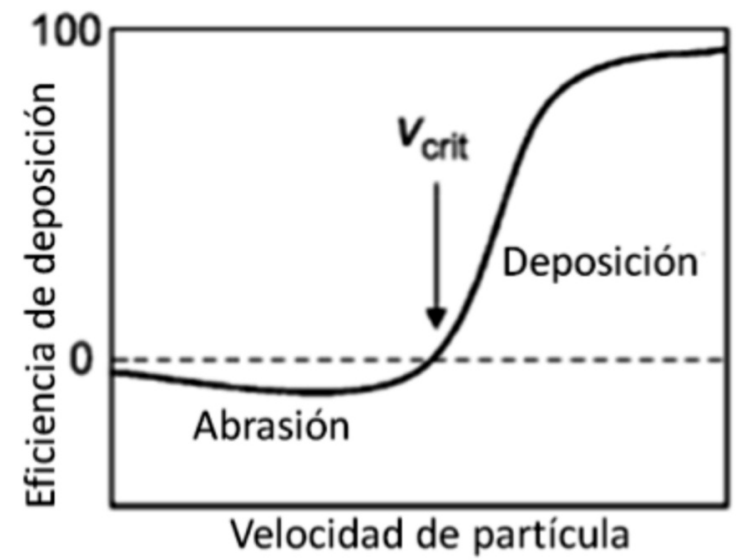

Figura 3. Efecto de la velocidad crítica en la eficiencia de la deposición.

Figure 3. Effect of the critical velocity on deposition efficiency. 


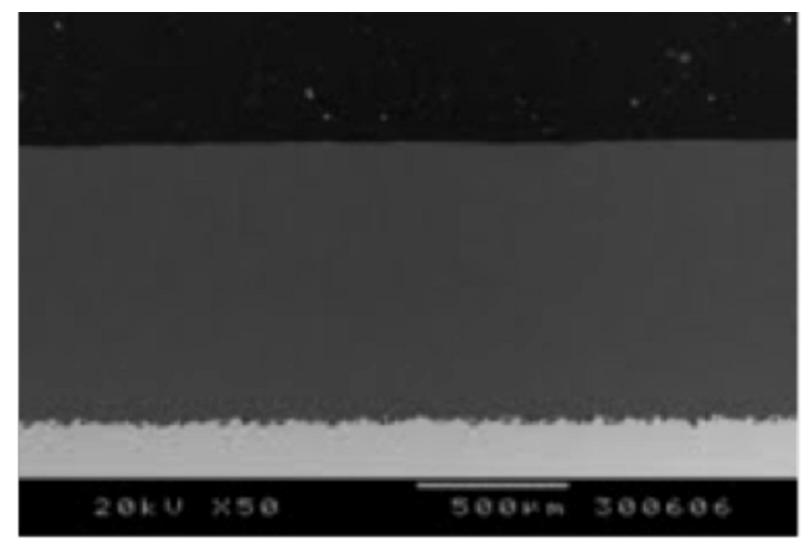

Figura 4. Micrografía SEM de un recubrimiento de aluminio sobre acero obtenido por CGS ${ }^{[20]}$.

Figure 4. The SEM micrograph of cold sprayed aluminium coating on steel substrat ${ }^{[20]}$.

\section{LAS ALEACIONES LIGERAS EN LA PROYECCIÓN FRÍA}

La necesidad de eficiencia energética en sistemas de transporte demanda el uso de aleaciones ligeras, siendo las aleaciones de base aluminio las predominantes. Los componentes de estas aleaciones están sometidos a procesos de corrosión y desgaste que los deterioran, exigiendo su substitución. El desgaste y la corrosión son dos de las principales causas de la degradación de los materiales y el fallo de productos y componentes de piezas industriales, lo cual implica discontinuidad en la operación de maquinaria, rotura de vehículos o paradas de líneas de producción, además de un elevado coste de reparación y mantenimiento. Un reciente informe presentado por NACE (National Association of Corrosion Engineers) ha estimado que en Estados Unidos, durante el año 2012, el costo anual causado por la corrosión excede el 6,2 \% del Producto Interno Bruto (PIB).

Debido a su alta resistencia y su bajo peso, las aleaciones de magnesio, representan una alternativa prometedora a las aleaciones de aluminio. Así, el magnesio de baja aleación ya ha reemplazado otros metales y un número elevado de plásticos en componentes de automóviles y camiones livianos. Se pueden citar como ejemplos cubiertas de válvulas y engranajes de distribución, bridas, bastidores de cajas de transmisión y embragues, radiadores, accesos de lámparas, carcasas de motores de limpiaparabrisas, y varias partes de reguladores interiores ${ }^{[21]}$.

La densidad específica del magnesio es un $35 \%$ inferior a la del aluminio, y típicamente sus aleaciones permiten una reducción de peso del $25 \%$ respecto a sus contrapartidas de aluminio ${ }^{[22]}$. Sin embargo, el magnesio es el más anódico de todos los metales estructurales, con un potencial de $-2,363 \mathrm{~V}$ respecto al electrodo de hidrógeno ${ }^{[23]}$. Por tanto, el magnesio tiene una baja resistencia a la corrosión lo que ha restringido la aplicación de sus aleaciones sin protección en entornos agresivos. La extrema susceptibilidad del magnesio y sus aleaciones respecto a la corrosión galvánica, puede ser mejorada mediante la aplicación de tratamientos superficiales ${ }^{[24]}$. Los métodos tradicionales presentan inconvenientes. El cromado por ejemplo puede suponer la exposición de los trabajadores a materiales peligrosos, como el cromo hexavalente, cuya normativa de uso se endurece cada día. Además, aun con este tratamiento superficial, las piezas en servicio tienen problemas de corrosión.

Las aleaciones de magnesio de la serie $\mathrm{AZ}$ pueden ser soldadas mediante técnicas TIG y MIG, aunque presentan zonas afectadas por el calor y distorsiones, por lo que deben realizarse aportes por micro-TIG para evitar estos problemas, aunque aun así las aleaciones $\mathrm{AZ}$ son proclives a la corrosión por tensión en las zonas afectadas en la soldadura, lo que no hace adecuada esta técnica para aplicaciones con elevadas tensiones dinámicas ${ }^{[25]}$.

La aplicación de recubrimientos mediante proyección fría de materiales con mejor comportamiento a la corrosión, como es el caso de algunas aleaciones de aluminio, representa una oportunidad tecnológica viable, ya que, para el remachado de componentes de magnesio sólo están recomendados los remaches dúctiles de aluminio, preferiblemente aleación 5056-H32, para minimizar la posibilidad de falla por corrosión galvánica ${ }^{[26]}$.

\subsection{Recubrimientos de aluminio y sus aleaciones por proyección fría}

Dado que la adherencia tiene lugar por deformación plástica, materiales ideales para la obtención de recubrimientos mediante proyección fría son aquellos metales con empaquetado de alta densidad y estructura cristalina cúbica centrada en las caras como es el caso del aluminio, cobre y níquel ${ }^{[9}$ y 20$]$.

En la última década los investigadores han concentrado sus esfuerzos en estudiar y comprender todos aquellos fenómenos involucrados en la obtención de recubrimientos mediante la técnica de proyección fría, tales como los mecanismos de adherencia partícula/sustrato-partícula/partícula ${ }^{[27]}$, los efectos de la geometría de la pistola de proyección ${ }^{[28]}$, la influencia de la naturaleza del sustrato en las características del recubrimiento ${ }^{[29]}$, el efecto de la morfología y distribución granulométrica del polvo en el comportamiento cinético de las partículas y mecanismos de adherencia $^{[30]}$ y todos aquellos factores que permitan a la comunidad científico-técnica comprender y opti- 


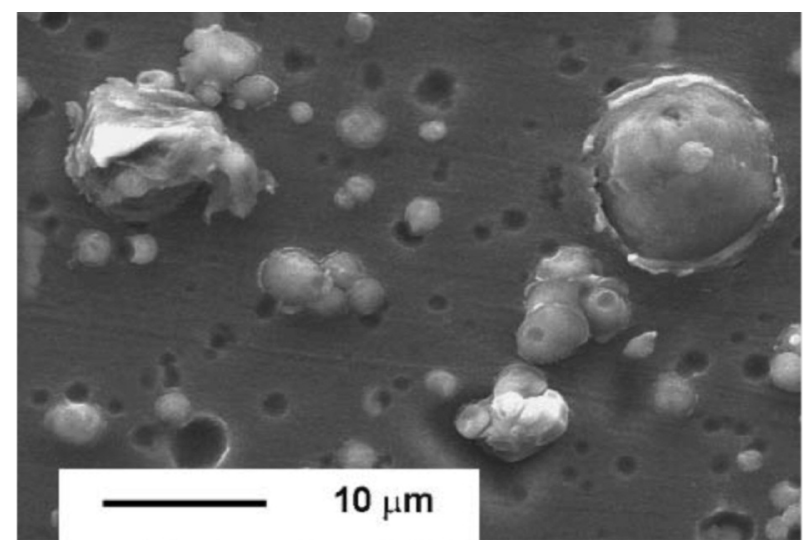

Figura 5. Partículas de Al proyectadas por proyección fría sobre sustrato de aleación de aluminio[31].

Figure 5. Al particles projected by cold spray onto aluminium alloy substrate ${ }^{[31]}$.

mizar los parámetros para la obtención de recubrimientos que cumplan las especificaciones requeridas.

En el caso del aluminio y sus aleaciones, las investigaciones han realizado contribuciones importantes que han permitido optimizar y adaptar dichos recubrimientos en función de las necesidades industriales actuales. Por ejemplo D. Zhang et al. ${ }^{[29]}$ han obtenido recubrimientos de aluminio sobre sustratos de diferente naturaleza, que incluye metales de diferentes durezas, polímeros y cerámicos, todos con una rugosidad inicial $\mathrm{Ra}<1 \mu \mathrm{m}$ con el fin de evaluar su influencia en las propiedades de los recubrimientos. Los resultados indican que el inicio de la deposición depende en gran medida del tipo de sustrato, obteniendo un máximo rendimiento con los aceros. Además detectaron deformación en los sustratos de más baja dureza (polímeros y aleación de aluminio) como consecuencia del fuerte impacto de las partículas.

En la micrografía de la figura 5 es posible apreciar las huellas que han dejado sobre el sustrato de aluminio las primeras partículas que no han quedado adheridas, así como aquéllas que sí lo han hecho. Esto concuerda con el planteamiento de Tokarev ${ }^{[31]}$, quien sugiere que las primeras partículas en impactar sobre el sustrato, en la técnica de proyección fría, juegan un papel activador de la superficie aportando una rugosidad adecuada para favorecer el apilamiento de las otras partículas.

Las aleaciones de aluminio con alto contenido de silicio presentan una alta resistencia al desgaste, bajo coeficiente de expansión térmica, buena soldabilidad y buena resistencia a la corrosión incluso en ambientes marinos. En la figura 6, se puede observar cómo el sistema binario Al-Si forma un eutéctico a una temperatura de $577^{\circ} \mathrm{C}$ y a una composición de $12,6 \%$ de $\mathrm{Si}$, donde la microestructura está formada por una matriz de fase $\alpha$ y una dispersión de fase $\beta$ que corresponde al silicio ${ }^{[32]}$.

La obtención de recubrimientos de Al-12Si (aleación de composición cercana a dicho punto eutéctico), ha sido especialmente investigada en el campo de la proyección fría en los últimos años.

El comportamiento de las partículas de $\mathrm{Al}-12 \mathrm{Si}$ al impactar sobre un sustrato de acero ha sido estudiado por J. Wu et al. ${ }^{[33]}$ demostrando que al aumentar

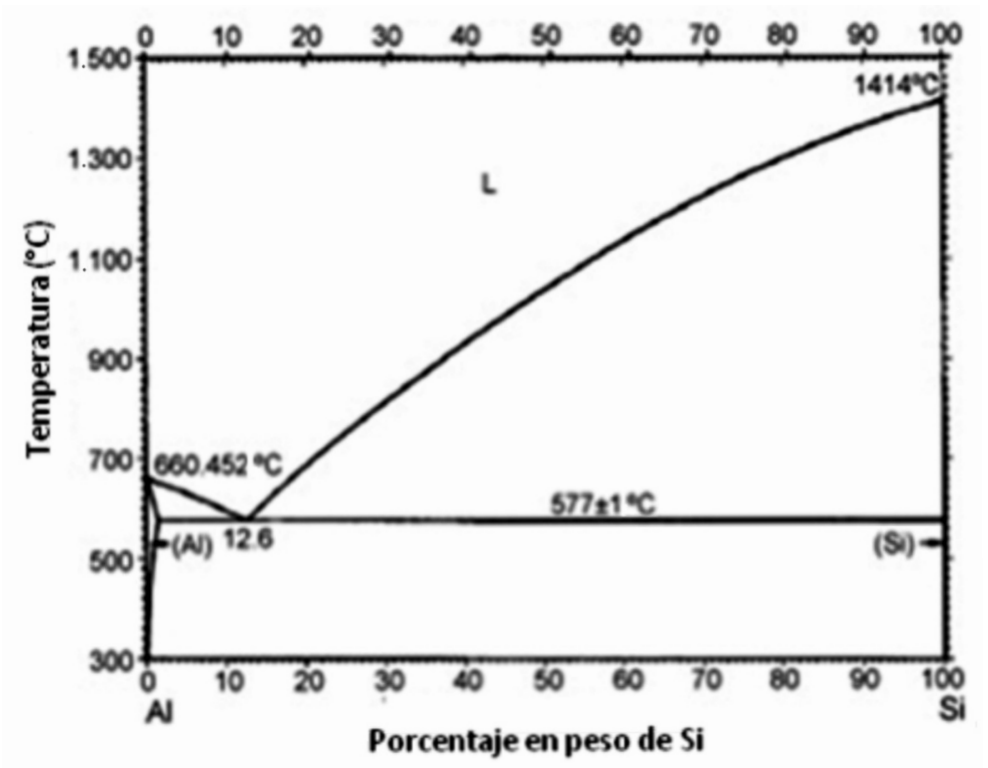

Figura 6. Diagrama de fases Al-Si.

Figure 6. The Al-Si phase diagram. 
la velocidad de las partículas el porcentaje de partículas adheridas tiene un aumento significativo, pero por encima de una velocidad de $700 \mathrm{~m} / \mathrm{s}$ este porcentaje comienza a disminuir (Figs. 7 y 8 ) y tiene lugar el inicio del rebote de las partículas.

Por su parte, L. Zhao et al. ${ }^{[34]}$ han investigado la influencia de los diferentes parámetros de proyección en la microestructura de los recubrimientos de $\mathrm{Al}-12 \mathrm{Si}$ sobre A16063, donde concluyen la importancia del control de la temperatura del gas ya que un aumento en la temperatura del gas ha representado un aumento en el espesor del recubrimiento obtenido, pero por otro lado un aumento en la porosidad; dichos resultados se presentan en la figura 9 .
Estos resultados han sido coherentes con los resultados obtenidos por W.Y. Li et al. ${ }^{[35]}$ quienes confirman el aumento de la porosidad con el aumento de la temperatura y además destacan el incremento de la dureza del recubrimiento $\left(99 \pm 12 \mathrm{HV}_{0,05}\right)$ con respecto a la dureza del polvo de partida $\left(64 \pm 8 \mathrm{HV}_{0,05}\right)$. Este aumento de los valores de dureza en recubrimientos obtenidos por proyección fría se debe principalmente a la severa deformación plástica que sufren las partículas al impactar con el sustrato; en casos como el níquel, estudios han revelado un incremento de un $66 \%$ respecto a la dureza del polvo inicial, para el acero inoxidable un $33 \%$ y para el aluminio cerca de un $48 \%{ }^{[36]}$.

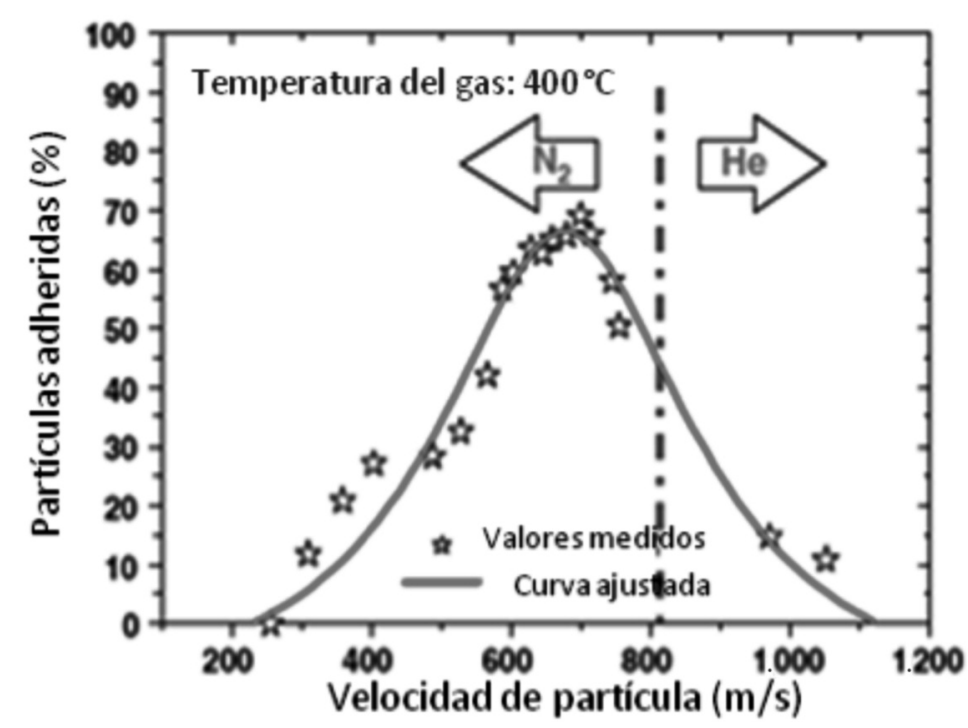

Figura 7. Relación entre la velocidad de partícula y porcentaje de partículas adheridas, para un polvo de Al-Si impactando sobre un sustrato de acero[33].

Figure 7. Relationship between particle velocity and ratio of bonds for an Al-Si feedstock impacting onto a mild steel substrate ${ }^{[33]}$.
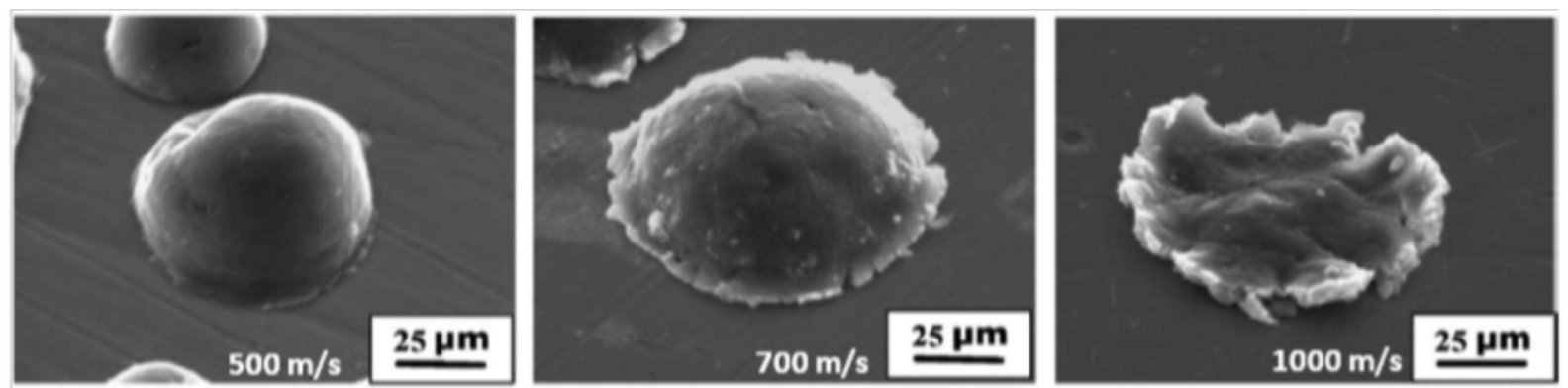

Figura 8. Micrografías SEM de partículas de Al-Si luego de impactar a diferentes velocidades sobre sustrato de acero[33].

Figure 8. SEM micrographs of Al-Si particles impacting at different velocity onto mild steel substrate ${ }^{[33]}$. 


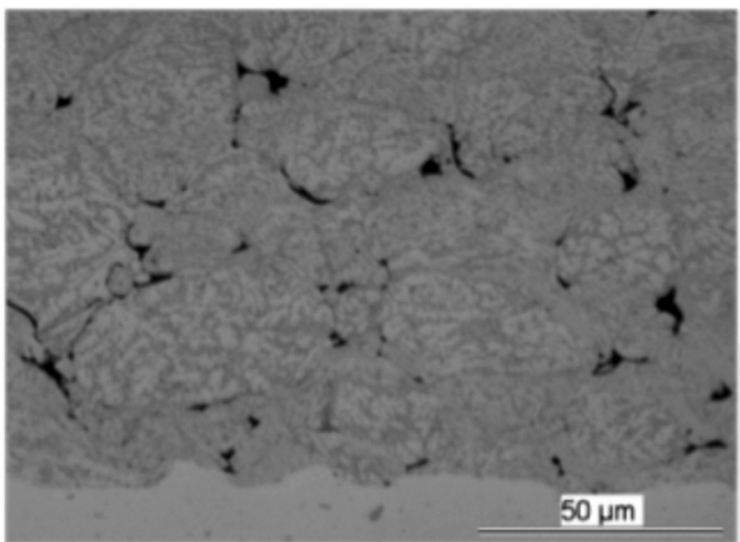

(a)

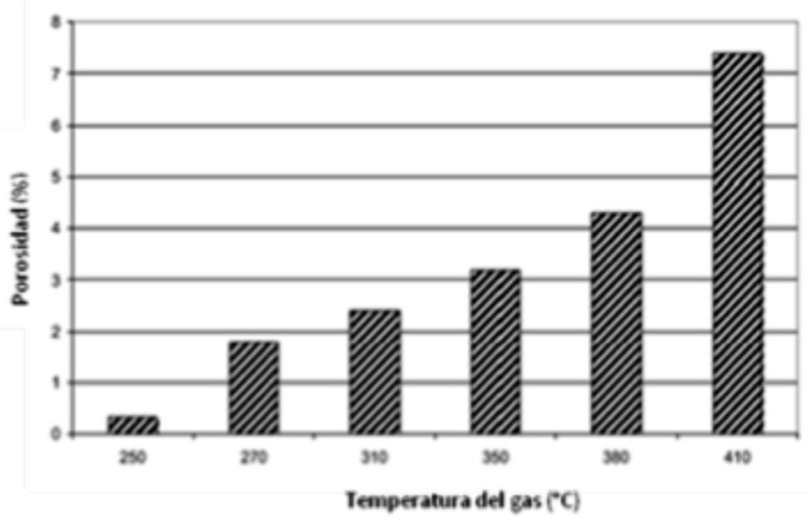

(b)

Figura 9. (a) Microestructura de un recubrimiento de Al-12Si depositado a una temperatura de gas de $350^{\circ} \mathrm{C}$; (b) relación de la temperatura del gas con la porosidad del recubrimiento ${ }^{[34]}$.

Figure 9. (a) Al-12Si coating deposited with a process gas temperature of $350^{\circ} \mathrm{C}$; (b) coating porosity in relationship to the process gas temperature ${ }^{[34]}$.

\subsection{Recubrimientos nano/micro- estructurados obtenidos por proyección fría}

Gracias a las múltiples investigaciones desarrolladas en los últimos años ${ }^{[37-43]}$ que ponen en evidencia la superioridad de las propiedades de los materiales en escala nanométrica y nanoestructurados con respecto a sus homólogos en escala micrométrica, no han tardado en surgir estudios para lograr obtener recubrimientos nanoestructurados por proyección fría.

Los materiales nano-estructurados exhiben una mayor resistencia mecánica, una alta tenacidad y mejo- res propiedades frente a la fatiga; asimismo, pueden comportarse súper-plásticamente a temperaturas relativamente bajas $(<0,5 \mathrm{Tm})$ y altas velocidades de deformación. Pero también es evidente que su ductilidad disminuye a baja temperatura, como se demuestra en los estudios del comportamiento del aluminio realizados por M.A. Haque ${ }^{[44]}$ y que se representa en la figura 10.

Para lograr obtener recubrimientos nanoestructurados, surgen nuevos retos para los investigadores: por una parte obtener el método y los parámetros de procesamiento que permitan obtener un tamaño de partícula y una estructura en la escala nanométrica, por otra parte redefinir los parámetros de proyección

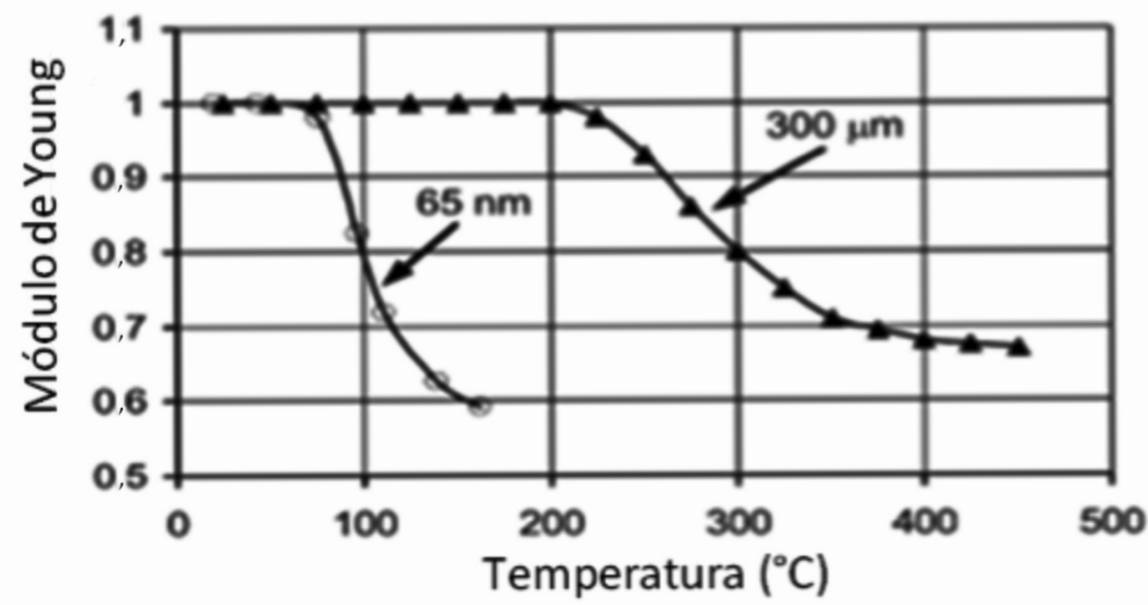

Figura 10. Comparación del módulo de Young a varias temperaturas para dos tamaños de grano diferentes de aluminio ${ }^{[44]}$.

Figure 10. Comparison of Young's modulus at various temperatures for two different grain sizes of aluminium ${ }^{[44]}$. 
ya que el tamaño de la partícula influye de manera directa en su comportamiento al ser proyectada ${ }^{[43]}$.

Una de las técnicas de procesamiento para reducir el tamaño y las características estructurales del polvo es la molturación criogénica (cryomilling) ${ }^{[45-54]}$. Esta técnica es una adaptación de la molturación convencional en la que el material es molturado en presencia de nitrógeno líquido; esta baja temperatura evita la recuperación y recristalización de las partículas, permitiendo obtener estructuras de grano más finas y reducción de tamaño más rápidamente ${ }^{[49]}$. En la molturación criogénica o crio-molturación, la formación de la estructura cristalina se da en tres etapas:

- Alta densidad de dislocaciones.

- Eliminación y nueva combinación de las dislocaciones.

- Transformación de estructura de límite de subgrano a límites de grano de ángulos definidos.
Estas etapas se representan en la figura 11.

L. Ajdelsztajn et al. ${ }^{[53]}$ han procesado mediante criomolturación polvo de alumino el cual ha sido posteriormente utilizado para obtener recubrimientos nanoestructurados por proyección fría. Sus resultados han demostrado que el proceso de criomolturación ha sido eficaz para reducir el tamaño y obtener una estructura cristalina, pero sin embargo los recubrimientos obtenidos a partir de este polvo, a pesar de tener una estructura cristalina, no presentan la densidad deseada. Sin embargo, Zhang et al. ${ }^{[54]}$ realizan nuevos ensayos a partir del mismo material pero variando el gas utilizado, nitrógeno en lugar de helio, y obtiene bajo las mismas condiciones de proyección unos resultados más satisfactorios. La grafica de la figura 12 , hace una comparación de los resultados obtenidos con ambas condiciones experimentales y muestra cómo la dureza del material aumenta al pro-
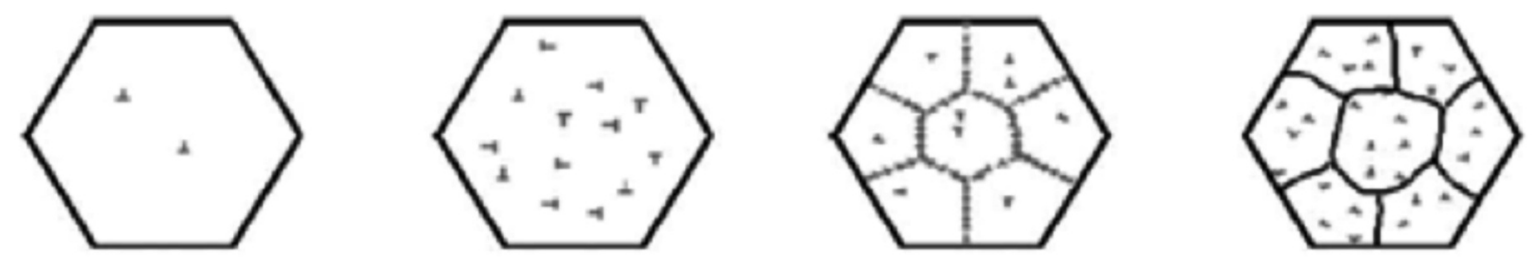

Incremento en el tiempo de moltura

Figura 11. Esquema del refinamiento del grano en el proceso de molturación criogénica[49].

Figure 11. A schematic illustration of grain size refinement during cryomilling ${ }^{[49]}$.

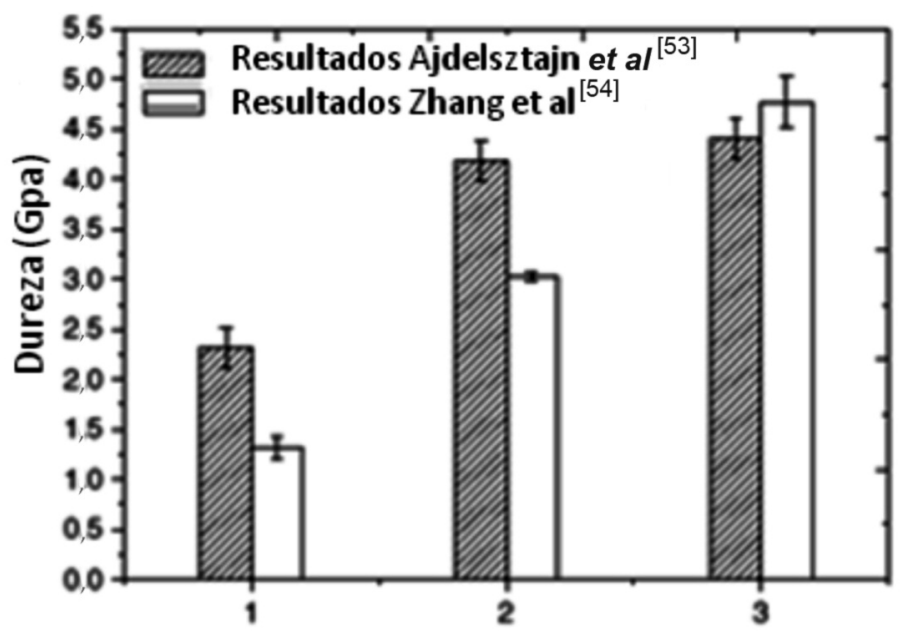

Figura 12. Comparación de la dureza del aluminio en tres fases diferentes; 1: polvo atomizado; 2: polvo crio-molturado; 3: recubrimiento por proyección fría ${ }^{[54]}$.

Figure 12. Comparison of the hardness of aluminum in three different phases; 1: as-atomized powder; 2: cryomilled powder; 3: al coating by cold spray ${ }^{[54]}$. 
cesarse por criomolturación e incrementa aún más al obtenerse el recubrimiento.

\section{APLICACIONES DE RECUBRIMIENTOS BASE ALUMINIO OBTENIDOS POR PROYECCIÓN FRÍA (CS)}

\subsection{Soldadura fuerte empleando proyección fría}

La soldadura fuerte, más conocida en terminología inglesa como brazing, es un proceso de unión térmica en el que el metal de aporte se funde a una temperatura por encima de los $450^{\circ} \mathrm{C}$ pero siempre por debajo del punto de fusión de los metales base a ser soldados ${ }^{[55]}$. Este proceso se realiza ya sea bajo atmósfera controlada (vacío) o más frecuentemente con el uso de fundentes (flux) que son aplicados a los metales base para protegerles de la formación de óxido durante el calentamiento y para favorecer el mojado del metal de aporte. Sin embargo la opción de la atmósfera controlada mediante vacío genera mayores costos y la segunda opción genera residuos que pueden ser corrosivos, lo que obliga a una exhaustiva limpieza de la superficie tratada para garantizar la calidad de la unión ${ }^{[56]}$.

En la soldadura fuerte de aluminio, el principal inconveniente es la formación de la capa de alúmina superficial y su gran estabilidad hasta temperaturas elevadas. La presencia de esta capa de óxido resulta perjudicial para la formación de una unión resistente ya que reduce la mojabilidad debido a su naturaleza cerámica y evita el contacto necesario entre el metal de aporte y el material base, por lo cual en este caso la utilización de fundente o una atmósfera controlada es necesaria para evitar la formación de más productos de oxidación durante el proceso de soldadura y eliminar, al menos en parte, los ya formados.

Otro inconveniente en el momento de soldar aleaciones de aluminio, es la susceptibilidad que tiene este tipo de aleaciones al agrietamiento en caliente. Para combatir este problema los soldadores aumentan la velocidad de la soldadura para reducir el aporte de calor ${ }^{[55]}$ o realizan un precalentamiento para disminuir el gradiente de temperatura a través de la zona de soldadura, lo cual ayuda a reducir el agrietamiento en caliente, pero puede modificar las características mecánicas del material base.

Zhao et al. ${ }^{[56]}$ estudiaron la viabilidad de utilizar la técnica de proyección fría sobre las superficies a unir, como un pre-tratamiento superficial para favorecer su posterior unión térmica mediante soldadura fuerte (brazing). Para ello depositaron una aleación de Al-12Si mediante proyección fría sobre sustratos de AA6063 (Fig. 13 (a)); algunas de las superficies recubiertas fueron después tratadas térmicamente a $500{ }^{\circ} \mathrm{C}$ y $615^{\circ} \mathrm{C}$ y posteriormente soldadas, obteniendo una unión en donde los límites entre el recubrimiento y el sustrato habían desaparecido a causa de la difusión generada por el proceso de soldadura, tal como se puede ver en la figura 13 (b). Los resultados revelan que este pre-tratamiento mediante proyección fría resulta favorable para la unión de las superficies mediante soldadura fuerte, dado que dicha técnica es un proceso que se lleva a cabo en estado

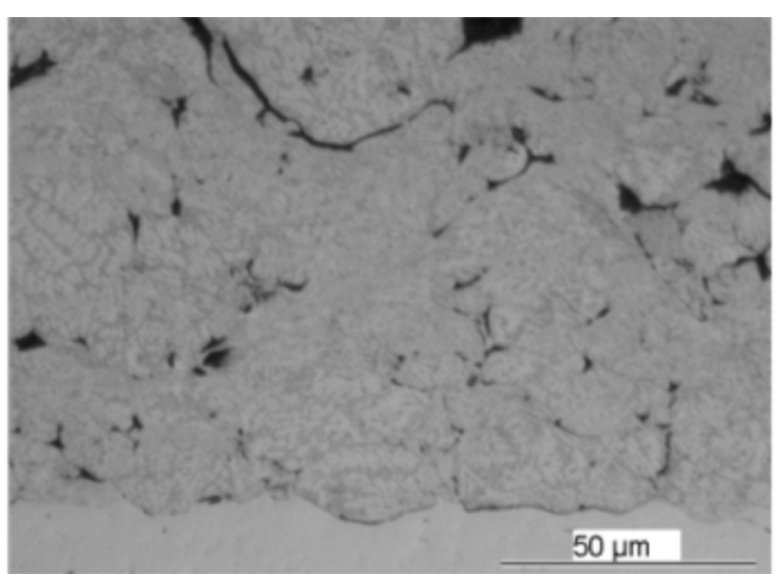

(a)

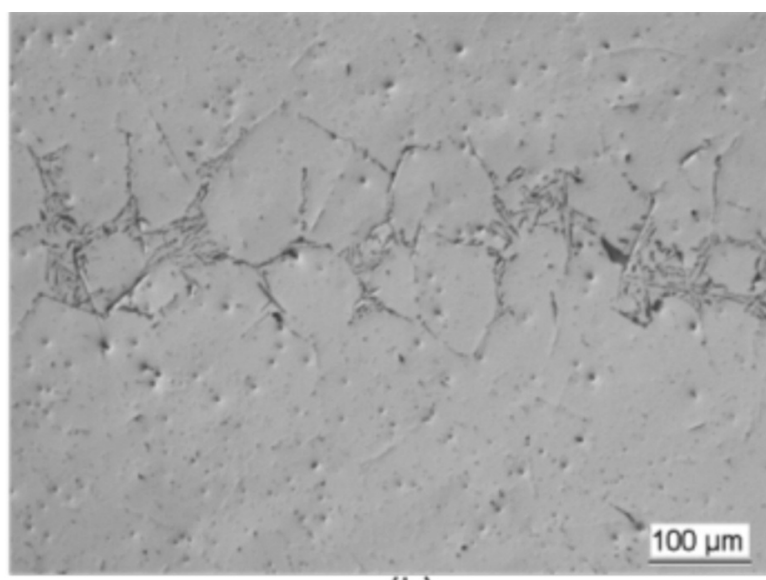

(b)

Figura 13. (a) Recubrimiento de Al-12Si sobre AA6063 pulida; (b) muestras recubiertas y luego soldadas (brazed) utilizando una atmósfera de argón ${ }^{[56]}$.

Figure 13. (a) Al-12Si coating on polished AA6063; (b) a sample brazed under argon atmosphere ${ }^{[56]}$. 
sólido con el cual se elimina el problema de oxidación; además, las altas velocidades con las que impactan las partículas sobre la superficie hacen que se rompan las capas de óxido presentes tanto en la superficie del sustrato como de las partículas, logrando así mejorar la mojabilidad de las superficies al aplicar el material de aporte por soldadura fuerte y por ende mejorar la calidad de la unión.

En una publicación más reciente, Bobzin et al. ${ }^{[57]}$, utilizan aleaciones de $\mathrm{Al}-7 \mathrm{Si}, \mathrm{Al}-12 \mathrm{Si}$ y $\mathrm{Al}-12 \mathrm{Si} 4 \mathrm{Cu}$ para tratar las superficies por proyección fría de placas de aluminio puro de $1,5 \mathrm{~mm}$ de espesor a unir por soldadura fuerte y así evaluar la posibilidad de no emplear fundentes durante la soldadura; los mejores resultados con y sin fundentes se obtuvieron con las superficies tratadas previamente por proyección fría con la aleación de Al-12Si (Fig. 14).

\subsection{Restauración de componentes industriales}

La industria y la comunidad científica están poniendo especial énfasis en el desarrollo de aplicaciones para la prevención de la corrosión y el aumento de la vida útil en aleaciones ligeras.

Algunas de las características más representativas de los recubrimientos obtenidos por proyección fría son su bajo contenido de oxígeno, baja porosidad, la presencia de tensión residual mayoritariamente a compresión, el no crecimiento de los granos ni transformaciones de fase ${ }^{[58]}$. Estas propiedades hacen esta técnica ideal para tratar aquellos materiales altamente susceptibles a cambios estructurales por la temperatura empleada en otros procesos tales como la soldadura.

La restauración de componentes de elevado valor industrial es una de las múltiples aplicaciones de esta técnica, como es el caso de la restauración de la pieza de un motor de una aeronave con severo daño por corrosión (Fig. 15), donde la superficie es reparada aplicando un recubrimiento de aluminio por proyección fría y posteriormente mecanizada para conservar sus dimensiones ${ }^{[59]}$. El laboratorio de materiales del departamento de defensa americano presenta varios casos como éstos, con los cuales se pone en evidencia las ventajas técnicas y económicas de la proyección fría ${ }^{[60 \text { y } 61]}$.

Componentes como éstos son comúnmente fabricados con aleaciones ligeras de aluminio y magnesio, las cuales se ven frecuentemente afectadas por problemas de corrosión y desgaste. Reparar por otras técnicas de proyección térmica e incluso por soldadura puede alterar las propiedades de los materiales y resultar menos favorable.

Otro amplio campo de aplicación es la reparación de moldes utilizados para la conformación de piezas en la industria del plástico, ya que las fuerzas mecánicas y altas temperaturas involucradas en el proceso desgastan el molde además de generar corrosión a causa de la fatiga térmica. Varias técnicas han sido utilizadas para la restauración de moldes, entre ellas la soldadura pero los esfuerzos residuales y la reparación repetitiva en partes localizadas disminuyen el tiempo de vida del molde reparado.

J. Lee et al $!^{[62]}$ han optado por emplear la técnica de proyección fría para reparar un molde fabricado en

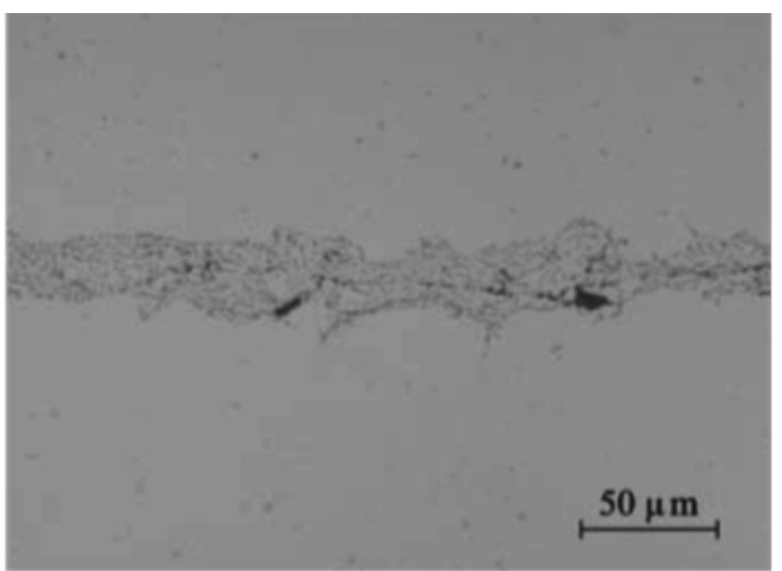

(a)

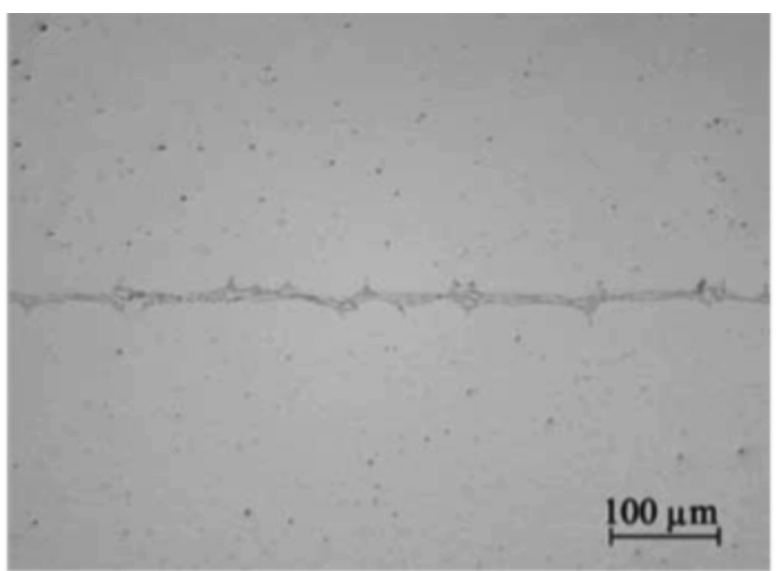

(b)

Figura 14. Sección transversal de la unión de dos placas mediante soldadura fuerte (brazing) con un recubrimiento de Al-12Si sobre las superficies a unir (a) sin emplear fundente durante la soldadura; (b) con fundente ${ }^{[57]}$.

Figure 14. Cross-section of a joint brazed with an Al-12Si-deposited sample (a) without fluxes; (b) with fluxes ${ }^{[57]}$. 

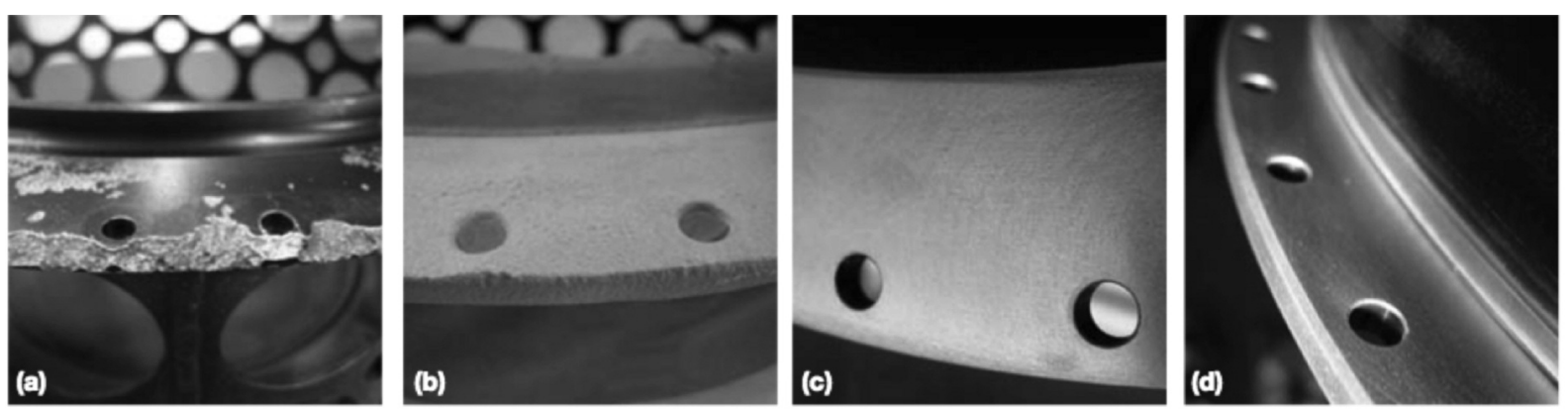

Figura 15. Reparación de un componente de una aeronave: (a) superficie corroída; (b) superficie tratada con proyección fría; (c) superficie maquinada; (d) pieza terminada ${ }^{[59] .}$

Figure 15. Repair of an aircraft component by cold spray: (a) before with extensive corrosion damage; (b) as sprayed with aluminium; (c) as machined; (d) finished part[59].

Al 6061-T6 utilizado para la inyección de piezas poliméricas; para la restauración han depositado partículas de aluminio puro sobre la superficie estropeada del molde y posteriormente se ha mecanizado hasta obtener las dimensiones y características propias del molde. Tras evaluar las propiedades mecánicas y comprobar la calidad de la intervención, se ha demostrado que el molde reparado presenta una mayor resistencia al desgaste.

Otra alternativa apta para mejorar las propiedades mecánicas de los componentes fabricados en aleaciones ligeras es mediante la aplicación de un recubrimiento compuesto metal-cerámico empleando la técnica de proyección fría. La figura 16 muestra cómo el coeficiente de desgaste de la sección de un molde reparado con un recubrimiento compuesto es menor respecto al coeficiente de desgaste del material original. Por su parte, recientes estudios desarrollados en el Centro de Proyección Térmica demuestran que este tipo de recubrimientos (matriz de bronce de aluminio reforzado con $\mathrm{Al}_{2} \mathrm{O}_{3}$ ) permite obtener recubrimientos con una resistencia al desgaste por deslizamiento cercana al $100 \%$ superior a la del bronce puro, mientras que la resistencia por abrasión mejora en más de un $30 \%{ }^{[63]}$. Alternativamente, estos investigadores han obtenido multiestructuras con recubrimientos de aluminio reforzados con alúmina en la capa superior, cuya dureza media superficial es tres veces superior a la del aluminio en estado de recocido $^{[20]}$; la microestructura de estos recubrimientos se puede ver en la figura 17 . Otros estudios empleando Inconel 625 reforzado con alúmina, han arrojado resultados igualmente satisfactorios ${ }^{[64]}$, lo que pone en evidencia las múltiples alternativas y campos de acción de la proyección fría.

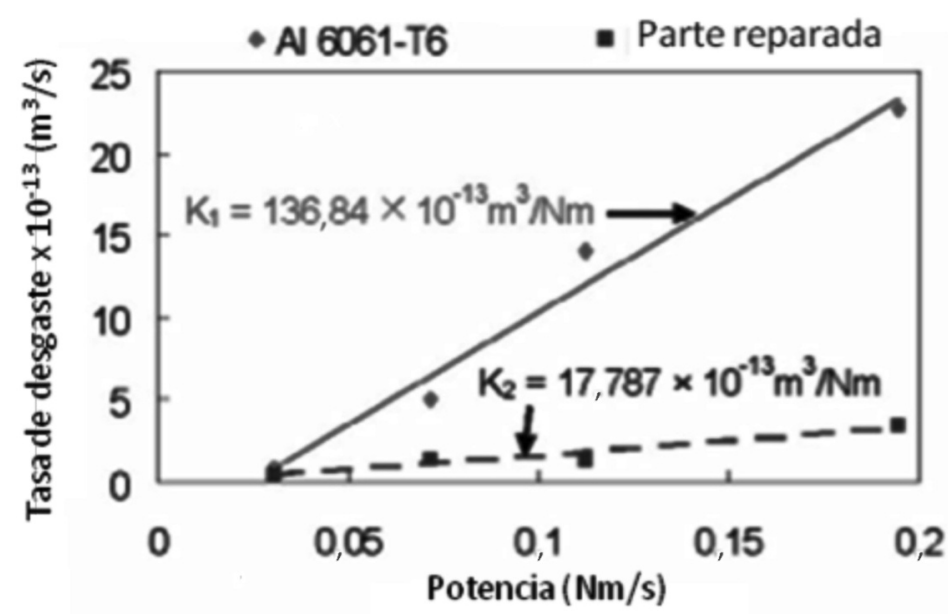

Figura 16. Coeficiente de desgaste del molde reparado con respecto al molde original Al 6061-T6 [62].

Figure 16. Wear coefficients of repaired specimen and original Al 6061-T6 [62]. 

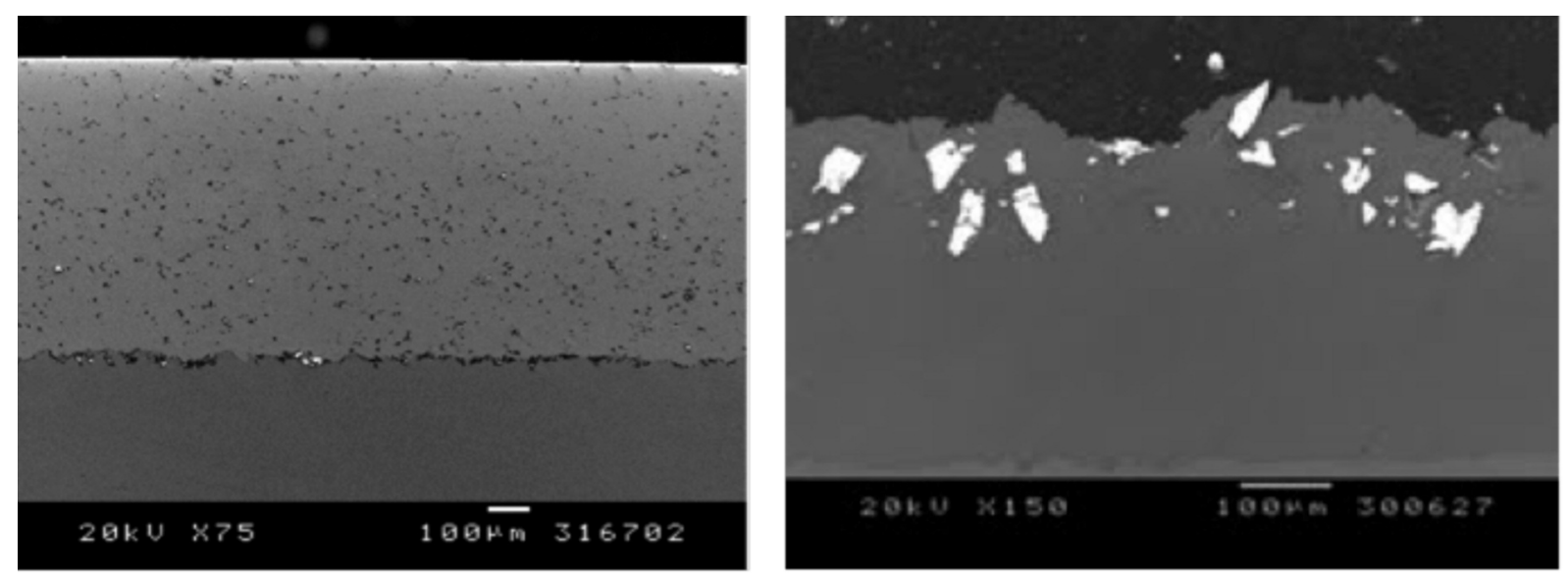

Figura 17. Micrografía SEM de recubrimientos obtenidos por proyección fría en el CPT: (a) bronce de aluminio reforzado con $3 \%$ de partículas de $\mathrm{Al}_{2} \mathrm{O}_{3}{ }^{[63]}$; (b) aluminio reforzado con $\mathrm{Al}_{2} \mathrm{O}_{3}$ en su capa superior ${ }^{[20]}$.

Figure 17. SEM micrograph of coatings obtained at CPT: (a) aluminium bronze reinforced with $3 \% \mathrm{Al}_{2} \mathrm{O}_{3}$ particles ${ }^{[63]}$; (b) aluminium reinforced with $\mathrm{Al}_{2} \mathrm{O}_{3}$ in the top layer ${ }^{[20]}$.

\section{CONCLUSIONES}

— Los diferentes estudios que se han realizado hasta la fecha permiten discernir que gracias a las propiedades de los recubrimientos de aluminio y sus aleaciones obtenidos mediante proyección fría, hacen posible considerar esta técnica como viable para extender la vida útil de los componentes de aleaciones ligeras como aluminio y magnesio, disminuyendo y simplificando los procesos de mantenimiento.

- Este hecho proporcionará nuevas oportunidades de utilización de estas aleaciones ligeras en la industria, en entornos agresivos y de mantenimiento crítico, y que se benefician de la utilización de materiales ingenieriles extremadamente livianos, como pueden ser los aerogeneradores y otros sectores, como el de las energías renovables, además de constituir alternativa a procesos como el AlClad en la industria aeronáutica, lo cual deja abierta una gran vía de investigación para la optimización de estos recubrimientos.

\section{Agradecimientos}

Los autores expresan su agradecimiento a la Generalitat de Catalunya proyecto SGR2009-390 y al Ministerio de Ciencia e Innovación MAT 2010-20311 por la financiación de este estudio. De igual manera se agradece al Centro de Proyección Térmica por la adju- dicación de la beca de investigación que ha permitido el desarrollo de este proyecto.

\section{REFERENCES}

[1] T. Sidhu, S. Prakash y R. Agrawal, Corros. Eng. Sci. Techn. 43 (2008) 335-342.

[2] R.C. Souza, H. Voorwald y M. Cioffi, Surf. Coat. Tech. 203 (2008) 191-198.

[3] J.M. Guilemany, J. Fernández, A.V. Benedetti y J. Delgado, Proc. International Thermal Spray Conference, ASM Thermal Spray Society, Dusseldorf, Germany, 2002, pp. 894-899.

[4] J. Fernández, M. Gaona y J.M. Guilemany, J. Therm. Spray Techn. 16 (2007) 220-228.

[5] H. Herman, S. Sampath y R. McCune, Mater. Res. Bull. 25 (2000) 17-25.

[6] J.M. Guilemany, N. Cinca, J. Fernández y S. Sampath, J. Therm. Spray Techn. 17 (2008) 762-773.

[7] A.E. Segall, A.N. Papyrin, J.C. Conway Jr. y D. Shapiro, JOM 50 (1998) 52-54.

[8] E. Irissou, J.G. Legoux, A.N. Ryabinin, B. Jodoin y C. Moreau, J. Therm. Spray Techn. 17 (2008) 495-516.

[9] H. Assadi, F. Gärtner, T. Stoltenhoff y H. Kreye, Acta Mater. 51 (2003) 4.379-4.394.

[10] A.N. Papyrin, V.F. Kosarev, S.V. Klinkow y A.P. Alkhimov, Proc. International Thermal Spray Conference, Dusseldorf, Germany, 2002, pp. 380-384. 
[11] M. Hammerschidt y H. Kreye, Shock waves and high strain-rate phenomena in metals, Ed. M.A. Meyers, L.E. Murr, Plenum Press, New York, 1981, p. 961.

[12] T.H. Van Steenkiste, J.R. Smith y R.E. Teets, Surf. Coat. Tech. 154 (2002) 237-252.

[13] T.H. van Steenkiste, J.R. Smith y R.E. Teets, Surf. Coat. Tech. 111 (1999) 62-71.

[14] D.L. Gilmore, R.C. Dykhuizen, R.A. Neiser, T.J. Roemer y M.F. Smith, J. Therm. Spray Techn. 8 (1999) 576-582.

[15] M. Villa, S. Dosta, J. Fernández y J. M. Guilemany, Rev. Metal. 48 (2012) 175-191.

[16] R.C. Dykhuizen y M.F. Smith, J. Therm. Spray Techn. 7 (1998) 205-212.

[17] C.J. Li y W.Y. Li, Surf. Coat. Tech. 167 (2003) 278-283.

[18] R. Kapoor y S. Nemat-Nasser, Mech. Mater. 27 (1998) 1-12.

[19] J.F. Molinari y M. Ortiz, Int. J. Impact. Eng. 27 (2002) 347-358.

[20] S. Dosta, C. Lorenzana, N. Cinca y J. M. Guilemany, Soldadura y Tecnologías de Unión 126 (2011) 13-14.

[21] D.S. Mehta, S.H. Masood y W.Q. Song, J. Mater. Process. Tech. 155 (2004) 1.526-1.531.

[22] S. Das, JOM 55 (2003) 22-26.

[23] M.G. Fontana, Corrosion Engineering, 3rd ed., McGraw-Hill International Editions, 1996, p. 42.

[24] J.E. Gray y B. Luan, J. Alloy. Compd. 336 (2002) 88-113.

[25] X. Cao, M. Jahazi, J.P Immarigeon y W. Wallace, J. Mater. Process. Tech. 171 (2006) 188.

[26] K.B Armstrong, Int. J. Adhes. Adhes. 3 (1983) 37-52.

[27] W.Y. Li, C. Zhang, X. Guo, C.J. Li, H. Liao, y C. Coddet, Appl. Surf. Sci. 254 (2007) 517 526.

[28] W.Y. Li, H. Liao, H.T. Wang, C.J. Li, G. Zhang, y C. Coddet, Appl. Surf. Sci. 253 (2006) 708713.

[29] D. Zhang, P. Shipway, y D. McCartney, J. Therm. Spray Techn. 14 (2005) 109-116.

[30] X.J. Ning, J.H. Jang, y H.J. Kim, Appl. Surf. Sci. 253 (2007) 7.449-7.455.

[31] A.O. Tokarev, Met. Sci. Heat Treat. 38 (1996) 136-139.

[32] F. Gil, C. Aparicio y D. Rodrígues, Aleaciones ligeras, Ed. Edicions UPC, Barcelona. España, 2001, p. 251.

[33] J. Wu, H. Fang, S. Yoon, H. Kim, y C. Lee, Scripta Mater. 54 (2006) 665-669.
[34] L. Zhao, K. Bobzin, D. He, J. Zwick, F. Ernst y E. Lugscheider, Adv. Eng. Mater. 8 (2006) 264267.

[35] W.Y. Li, C. Zhang, X.P. Guo, G. Zhang, H.L. Liao, y C. Coddet, Appl. Surf. Sci. (2007) 7.124-7.130.

[36] V.K Champagne, D.J Helfritch, M.D Trexler y B.M Gabriel, Modell. Simul. Mater. Sci. Eng. 18 (2010) 1-8.

[37] R. Rofagha, R. Langer, A.M. El-Sherik, U. Erb, G. Palumbo y K.T. Aust, Scr. Metall. Mater. 25 (1991) 2.867-2.872.

[38] R.B. Intrui y Z. Szklarska-Smialowska, Corrosion 48 (1992) 398-403.

[39] E. Sikora, X.J. Wei, y B.A. Shaw, Corrosion 60 (2004) 387-398.

[40] M.M. Sharma y C.W. Ziemian, J. Mater. Eng. Perform. 17 (2008) 870-878.

[41] L. Liu, Y. Li y F. Wang, Electrochim. Acta. 54 (2008) 768-780.

[42] L. Liu, Y. Li y F. Wang, Electrochim. Acta. 53 (2008) 2.453-2.462.

[43] J. Bedoya, S. Dosta, N. Cinca y J.M. Guilemany, XII Congr. Nac. de Materiales, Universidad de Alicante, CD-Rom ISBN978-84-695-3316-1, Sociedad Española de Materiales, Alicante, España, 2012.

[44] M.A. Haque y M.T.A. Saif, Thin Solid. Films 484 (2010) 364-368.

[45] E.L. Dreizin, Prog. Energy Combust. Sci. 35 (2009) 141-167.

[46] C. Goujon, P. Goeuriot, M. Chedru, J. Vicens, J.L. Chermant, F. Bernard, J.C. Niepce, P. Verdier, e Y. Laurent, Powder Technol. 105 (1999) 328-336.

[47] C. Junsheng, C. Hua, C. Hanbin, Y. Bin, F. Jianzhong, y Z. Jishan, J. Univ. Sci. Technol. B. 14 (2007) 523-528.

[48] C.C. Koch, Nanostruct. Mater. 9 (1997) $13-$ 22.

[49] E.J. Lavernia, B.Q. Han, y J.M. Schoenung, Mater. Sci. Eng. A. 493 (2008) 207-214.

[50] W.Y. Li, G. Zhang, C. Zhang, O. Elkedim, H. Liao, y C. Coddet, J. Therm. Spray Techn. 17 (2008) 316-322.

[51] N.Cinca, E. Hurtado, I.G. Cano y J.M Guilemany, Rev. Metal. 47 (2011) 197- 204.

[52] F. Zhou, D. Witkin, S.R. Nutt, y E.J. Lavernia, Mater. Sci. Eng. A. 375 (2004) 917-921.

[53] L. Ajdelsztajn, J. Schoenung, B. Jodoin, y G. Kim, Metall. Mater. Trans. A 36 (2005) 657. 666.

[54] Y. Zhang, X. Wu, H. Cui, y J. Zhang, J. Therm. Spray Techn. 20 (2011) 1.125-1.132.

[55] F. Wendl y K.D. Wupper, J. Mater. Process. Tech. 24 (1990) 355-364. 
[56] L. Zhao, K. Bobzin, F. Ernst, J. Zwick, J. Roesing, y E. Lugscheider, Adv. Eng. Mater. 8 (2006) 751-753.

[57] K. Bobzin, L. Zhao, T. Schlaefer, y T. Warda, Front. Mech. Eng. Chin. 5 (2010) 256-260.

[58] V. Luzin, K. Spencer, y M.X. Zhang, Acta Mater. 59 (2011) 1.259-1.270.

[59] J. Villafuerte y D. Wright, ASM Thermal Spray Society., 5 (2010) 13-15

[60] P.F. Leyman y V.K. Champagne, U.S. Army Research Laboratory, ARL Report № ARLTR-4922, 2009, pp. 1-22.
[61] V.K. Champagne, P.F. Leyman, y D.J. Helfritch, U.S. Army Research Laboratory, ARL Report № ARL-TR-4438, 2008, pp. 1-26.

[62] J.C. Lee, H.J. Kang, W.S. Chu, y S.H. Ahn, CIRP Ann. Manuf. Techn. 56 (2007) 577-580.

[63] J. Miguel, S. Vizcaíno, S. Dosta, N. Cinca, C. Lorenzana y J.M Guilemany, Rev. Metal. 47 (2011) 390-401.

[64] S. Dosta, N. Cinca y J.M. Guilemany, International Thermal Spray Conference ITSC 2012, ASM Thermal Spray Society, Houston, EE.UU., 2012. 